\title{
Uso da terapia fotodinâmica empregando azul de metileno a $2 \%$ como agente fotossensibilizante para o controle de crescimento tumoral em calopsita (Nynphicus hollandicus) portador de sarcoma*
}

\author{
Use of photodynamic therapy using methylene blue $2 \%$ \\ as photosensitizing agent to control tumor growth \\ in a cockatiel (Nynphicus hollandicus) carrier sarcoma
}

\author{
Carolina Bistritschan Israel, ${ }^{* *}$ Daniel Federici Santos, ${ }^{* *}$ Tábata Maués, ${ }^{* \star}$ Gabriel Bobány de Queiroz, ${ }^{* \star}$ \\ Fabíola Barroso Costa, ${ }^{* *}$ Juliana Silva do Nascimento, ${ }^{* *}$ Sávio Freire Bruno, ${ }^{* *}$ Maria de Lourdes Gonçalves Ferreira**
}

\begin{abstract}
Resumo
Os sarcomas são neoplasias de origem mesenquimal, sendo considerados tumores agressivos que frequentemente geram metástases e rápida debilidade. A indicação terapêutica é a exérese tumoral enquanto ainda não houver metástases. A terapia fotodinâmica tem sido utilizada para o tratamento de neoplasias tanto em humanos quanto em animais e muitos estudos têm sido desenvolvidos de maneira a aprimorar e avaliar a efetividade da técnica. Uma calopsita (Nynphycus hollandicus) foi levada para atendimento no Hospital Veterinário Professor Firmino Mársico Filho (HUVET-UFF) apresentando nódulo em região escapuloumeral. O diagnóstico citopatológico concluiu sarcoma. Devido à localização e impossibilidade de exérese cirúrgica, optou-se pela realização da terapia fotodinâmica utilizando-se o azul de metileno a $2 \%$ como agente fotossensibilizador e fonte de luz laser diodo (Vetlight $($ ). . A terapia fotodinâmica empregando o azul de metileno como agente fotossensibilizante foi eficaz no controle do crescimento tumoral até os 30 dias posteriores observados.
\end{abstract}

Palavras-chave: aves, fotoquimioterapia, neoplasia.

\begin{abstract}
Sarcoma are tumors of mesenchymal origin. They are aggressive tumors that frequently metastasize and rapid weakness. The therapeutic indication is the resection while there is still no metastasis. Photodynamic therapy has been used for the treatment of cancer in both humans and animals and many studies have been developed in order to improve and enhance the effectiveness of the techniques. A Nynphycus hollandicus was brought to HUVET-UFF clinical service presenting a lump in scapulohumeral region. The citological exam had a diagnosis of sarcoma. Due to its location and inability to surgical excision, was decided by the completion of photodynamic therapy using methylene blue at $2 \%$ as a phosensitizing agent and laser diode light source (Vetlight $\circledast$ ). Photodynamic therapy using methylene blue as photosensitizing agent was effective in controlling growth up to 30 days later observed.
\end{abstract}

Keywords: birds, neoplasia, photochemotherapy.

\section{Introdução}

As aves de estimação podem apresentar ampla variedade de doenças neoplásicas (Araújo et al., 2007). As neoplasias são comumente detectadas nessas aves, em função de serem mais facilmente observadas, além de terem uma vida mais longa e uma predisposição genética que pode estar associada à endogamia (Latimer, 1994). A exposição a agentes carcinogênicos ambientais e nutricionais pode ser considerada um fator predisponente (Sinhorini, 2008). Linfomas, fibromas, fibrossarcomas, lipomas e lipossarcomas são alguns dos principais tumores observados (Reece, 1992).
Os sarcomas frequentemente apresentam-se como massas firmes e de difícil delimitação. A análise citopatológica é uma ferramenta de triagem que permite a detecção de células tumorais assim como do tecido de origem. A histopatologia é o método de eleição para o diagnóstico mais detalhado das massas tumorais (Withrow, 2007). O tratamento baseia-se na excisão radical antes da detecção de metástase, o que pode ocasionar sangramento extenso (Latimer, 1994).

Terapias alternativas como a fotodinâmica têm sido propostas no intuito de se evitar efeitos colaterais próprios de terapias tradicionalmente utilizadas no combate ao câncer (Martines et al., 2007).

\footnotetext{
*Recebido em 6 de novembro de 2013 e aceito em 30 de junho de 2014.

**Programa de Pós-graduação em Medicina Veterinária (Clínica e Reprodução Animal) da Universidade Federal Fluminense, Rua Vital Brazil ,64 - Niterói, RJ - CEP 24.230-340.

Autor para correspondencia: ccarolbia@ig.com.br
} 
Essa modalidade terapêutica tem como princípio uma reação fotoquímica (não térmica) que requer simultaneamente um fotossensibilizante, oxigênio e luz visível (Martines et al., 2007). Quando o composto fotossensível é introduzido no paciente, se acumula preferencialmente em células de rápida multiplicação. Então, ao serem irradiadas, ocorre ativação do composto gerando formas de oxigênio reativo (singlete e triplete), que agridem as células tumorais geralmente levando à morte e, consequentemente, resultando em ação curativa (Simplicio et al., 2002).

O azul de metileno é um fotossensibilizador com características desejáveis para o uso em terapia fotodinâmica, pois pode gerar altas concentrações de oxigênio singlete, que é a principal espécie de oxigênio responsável pela indução de morte celular na terapia. Além disso, o azul de metileno pode também induzir a formação de radicais que levam a danos em biomoléculas (Severino et al., 2003; Tardivo et al., 2002).

Em medicina veterinária, a terapia fotodinâmica tem sido aplicada em carcinomas de células escamosas de felinos, com êxito, utilizando-se fotossensibilizadores não fenotiazínicos (Emilio et al., 2006; Ferreira et al., 2009) porém ainda há muito a ser estudado, principalmente no que diz respeito a técnicas mais acessíveis que permitam o uso da técnica de forma mais frequente.

Este trabalho tem como objetivo relatar o uso da terapia fotodinâmica com azul de metileno em calopsita (Nynphycus hollandicus), portador de sarcoma, no controle do crescimento tumoral.

\section{Relato de caso}

Uma calopsita (Nynphycus hollandicus), macho, de 11 meses de idade, foi atendida no HUVET-UFF, cuja queixa principal do proprietário foi o aparecimento de nódulo em região escapuloumeral com crescimento contínuo. O animal apresentava comportamento normal, alimentava-se bem, e não apresentava outras alterações clínicas. O nódulo tinha consistência firme, aderido, não ulcerado, medindo 2,4 x 1,5 x $1,4 \mathrm{~cm}$ e com evolução em 20 dias.

Durante a palpação foram observados sinais de inflamação. Em função disso, administrou-se maxicam $(0,5 \mathrm{mg} / \mathrm{kg}$, s.i.d), durante 4 dias e, posteriormente, realizou-se punção por agulha fina para análise citológica. A análise citológica demonstrou tratar-se de neoplasia de origem mesenquimal (sarcoma). Radiografias torácicas (lateral e ventro-dorsal) foram realizadas, não evidenciando sinais de metástases visíveis.

A lesão encontrava-se na base da asa, não permitindo exérese cirúrgica por risco de lesão permanente, perda de função do membro e não obtenção de margem cirúrgica.

Procedeu-se a administração de cloridrato de tramadol (15 mg/ $\mathrm{kg} /$ t.i.d, VO) três dias antes da terapia fotodinâmica. Esta foi efetuada empregando o aparelho Vetlight $®$ (DMC goup), com comprimento de onda de 650-670 nm, potência de $1740 \mathrm{~mW}$ (através de 6 lâmpadas LEDs) e utilizou-se o corante azul de metileno a $2 \%$ como agente fotossensibilizante.
O animal foi submetido a anestesia geral que consistiu da indução e manutenção anestésica com isoflurano por meio de inalação em máscara (Figura 1). O Doppler vascular foi utilizado para monitoração do animal.

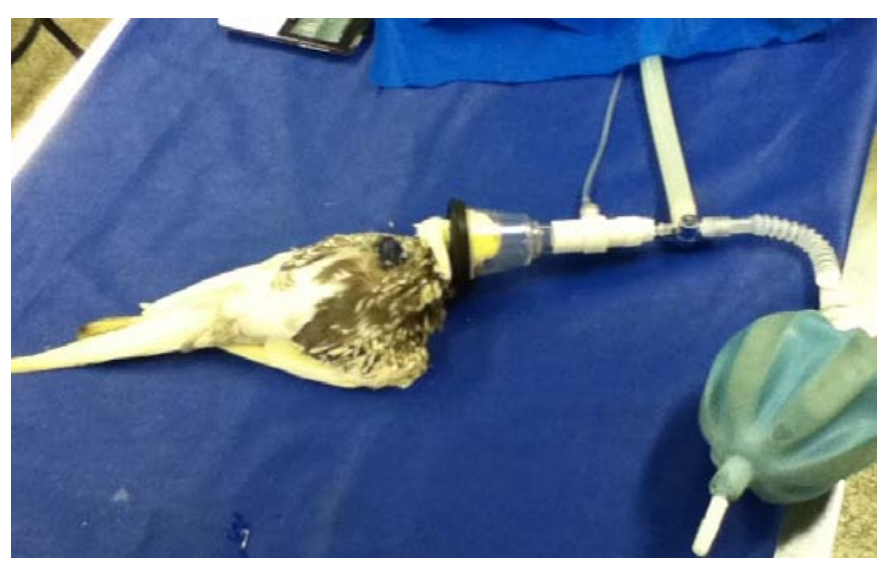

Figura 1: Ninphycus hollandicus sendo submetido a anestesia geral inalatória por inalação em máscara (isoflurano).

Com o animal anestesiado, procedeu-se infiltração intralesional de $0,3 \mathrm{ml}$ de azul de metileno a $2 \%$ utilizando-se agulha de tamanho $12,7 \mathrm{~mm} \times 0,33 \mathrm{~mm}$ (Figura 2), de forma que a maior parte do tumor adquirisse coloração azul intensa. Para delimitar a superfície a ser irradiada, utilizou-se tecido não tecido (TNT) que serviu para a proteção da ave, deixando apenas a lesão exposta à luz.

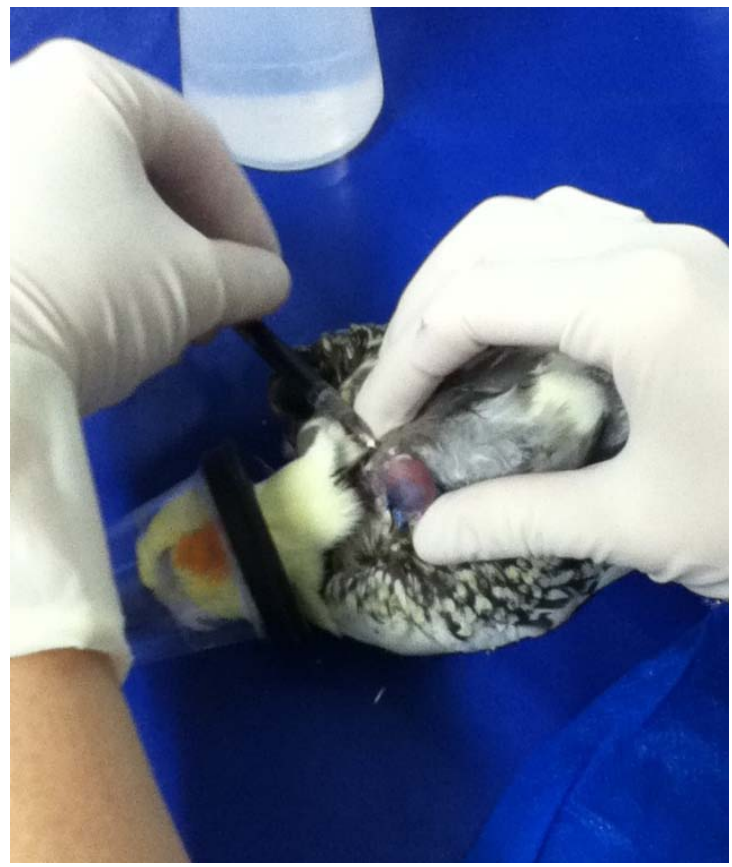

Figura 2: Infiltração intralesional de azul de metileno $(0,3 \mathrm{ml})$ com agulha tamanho $12,7 \mathrm{~mm}$ x 0,33 mm em Ninphycus hollandicus. 


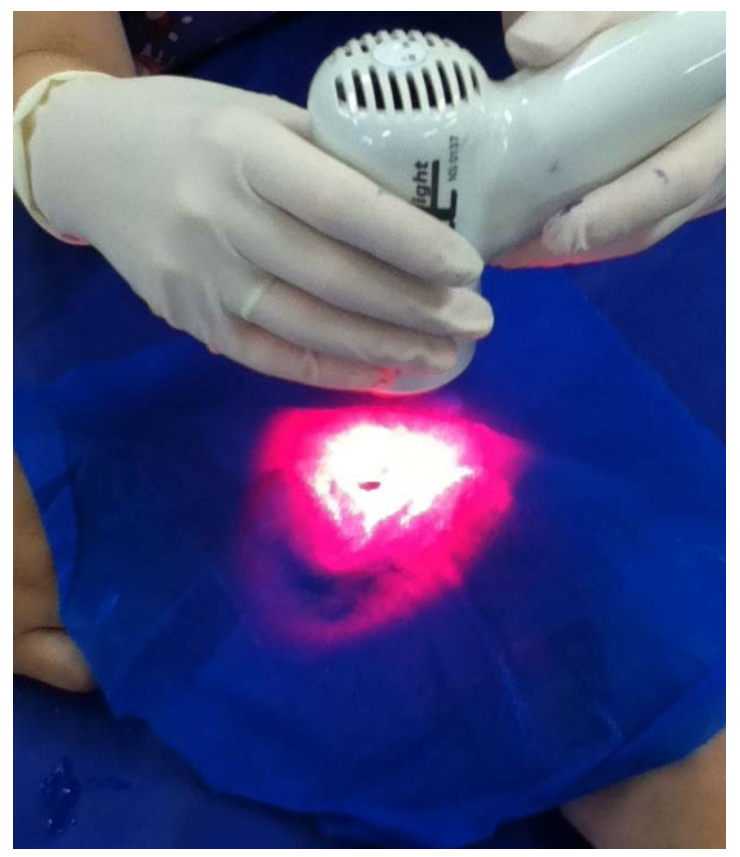

Figura 3: Lesão tumoral em Ninphycus hollandicus sendo irradiada empregando o equipamento Vetlight $\circledast$.

A lesão foi irradiada Figura 3) por 30 minutos a uma distância de $30 \mathrm{~cm}$. Durante o procedimento, não houve alterações de temperatura sensíveis ao tato. Nenhuma alteração foi observada na lesão durante o procedimento.

Prescreveu-se para o pós-operatório cloridrato de tramadol (15 $\mathrm{mg} / \mathrm{kg} /$ q.i.d.). Após sete dias, o animal retornou para revisão e a lesão encontrava-se ainda bem azulada e edemaciada. Foi então prescrito maxicam $(0,5 \mathrm{mg} / \mathrm{kg} / \mathrm{s}$.i.d.). Oito dias após o procedimento, a lesão encontrava-se com superfície crostosa e não havia aumentado de tamanho. $O$ animal não apresentou sinais de desconforto, mantendo-se ativo e alimentando-se bem nos 30 dias posteriores ao procedimento. Ao exame físico, observou-se estabilização da lesão, por não se observar aumento do nódulo após a terapia fotodinâmica. Após a retirada da crosta presente, as medidas tumorais eram semelhantes às primeiras $(2,3 \times 1,3 \times 1,4 \mathrm{~cm})$. Uma nova sessão de fototerapia seria realizada após 40 dias da primeira.

\section{Discussão}

A ave do presente relato apresentou lesão neoplásica maligna localizada na base da asa, o que está de acordo com o descrito por Latimer (1994), que refere que as neoplasias malignas em aves são duas vezes mais descritas que as benignas. O método diagnóstico empregado permitiu detectar-se a origem tumoral, mas não o tipo específico do mesmo, uma vez que para isto seria necessária a análise histopatológica (Withrow, 2007).

As neoplasias de origem mesenquimal estão relacionadas com as metástases e grave debilidade, fato que pode determinar a morte do animal antes mesmo de alguma tentativa de tratamento (Fernandéz-Bellon et al., 2013; Manarolla et al., 2008; Tavassoly, 2001; Maluenda et al., 2010). No presente relato, no entanto, o animal não apresentou debilidade ou metástases antes e durante o tratamento.

Para Latimer, 1994, a terapia indicada para os sarcomas é a exérese cirúrgica antes da ocorrência de metástases. No paciente em questão o tratamento cirúrgico foi impossibilitado pela localização tumoral.

A terapia fotodinâmica no tratamento de neoplasias tem sido utilizada com mais frequência no homem e em cães e gatos (Tardivo et al, 2002; Allison et al, 2010; Ferreira et al., 2006; Dagli et al, 2006; McCaw et al., 2010). Estudos laboratoriais têm sido desenvolvidos de modo a elevar a eficácia dessa modalidade terapêutica (Carvalho, 2008). Tendo em vista a impossibilidade de exérese cirúrgica instituiu-se a terapia fotodinâmica. A técnica, descrita em aves como o periquito de colar (Psittacula Krameri) (Suedmeyer et al., 2007), calau bicórnico (Buceros bicornis) (Suedmeyer et al., 2001; Miller et al., 1985) e calopsitas (Nynphicus hollandicus) (Rosenthal et al., 2001) já foi empregada para casos de carcinomas de células escamosas, porém, não em sarcomas.

De acordo com Tardivo et al. (2002), o azul de metileno temse mostrado um bom agente fotossensibilizador para o uso da terapia fotodinâmica com laser diodo, combinando eficácia e baixo custo. No presente relato, o azul de metileno foi considerado bom fotossensibilizante, com baixo custo e de fácil aplicação, não apresentando efeitos tóxicos ou alterações deletérias detectáveis clinicamente.

O uso da terapia fotodinâmica permitiu a realização de tratamento local sem efeitos colaterais sistêmicos, fato ressaltado por McCaw et al. (2010), ao afirmarem que a fototerapia não gera danos às regiões adjacentes e distantes à neoplasia. Os mesmos autores ressaltam a não ocorrência de resistência tumoral, diferente do que se observa na quimioterapia antineoplásica.

Diante do exposto, são necessárias maiores pesquisas acerca de terapias antineoplásicas em aves, tendo em vista que a exérese cirúrgica nem sempre é possível.

\section{Conclusão}

A terapia fotodinâmica empregando o azul de metileno como agente fotossensibilizante foi eficaz no controle do crescimento tumoral. 


\section{Referências}

ALLISON, R.R.; SIBATA, C.H.; Oncologic photodynamic therapy photosensitizers: A clinical review, Photodiagnosis and Photodynamic Therapy, v. 7, p. 61-75, 2010.

ARAÚJO, A.C.P.; CARVALHO, A.D.; NASCIMENTO, P.B.; VOLL, J.; DREIMMEIR, D.; Rabdomiossarcoma alveolar em papagaio (Amazona Aestiva); Acta Scientiae Veterinariae, v. 35, n. 1, p. 115-117, 2007.

CARVALHO, E.S.; Terapia fotodinâmica utilizando o cloridrato de aminolevulinato de metila no modelo de câncer bucal em murino induzido por células de Walker 256, Dissertação de mestrado, Faculdade de Ciências da Saúde da Universidade de Brasília, 2008.

DAGLI, E.C.R.; DUTRA, M.L.Z.; BECHARA, E.J.H.; GIOSO, M.A.; LARSSON, C.E.; PINOTTI, M.; ZEZELL, D.M.; Novo protocolo de terapia fotodinâmica utilizando-se o metil aminolevulinato e DTPA no tratamento de carcinoma espinocelular em felinos, XI Congresso Brasileiro de Física Médica, 2006.

EMILIO, C.R.; DAGLI, M.L.Z.; DUTRA, F.; BERCHARA, E.J.H.; GIOSO, M.A.; LARSSON, C.E.; PINOTTI, M.; ZEZELL,D.M.; A new protocol for photodynamic therapy using methyl aminolevulinate and DTPA in the treatmente of feline squamous cell carcinoma. Anais do IX Congresso de física médica, 2006.

Ferreira, I.; Rahal, S.C.; Ferreira, J.; Corrêa, T.P.; Terapêutica no carcinoma de células escamosas cutâneo em gatos. Ciência Rural, v. 36, n. 3, p. 1027-1033, 2006.

FERREIRA, I.; RAHAL, S.C., ROCHA, N.S.; GOUVEIA, A.H.; CORREA, T.P.; CARVALHO, Y.K.; BAGNATO, V.S.; Hematopotphyrin-based photodynamic therapy for cutaneous squamous cell caarcinoma in cats. Vet Dermatol, v. 20, n. 3, p. 174-178, 2009.

FERNÁNDEZ-BELLON, H.; MARTORELL, J.; RABANAL, R.; RAMIS, A.; Rhabdomyosarcoma in a Racing pigeon (Colomba Livia), Avian Pathology, v. 32, n. 6, p. 613-616, 2003.

FUJIMOTO, Y.; OKADA, K.; OKADA, M.; Rhabdomyossarcoma in the chicken, Jap. J. vet. Res, v.18, p. 109-115, 1970.

LATIMER, K.S.; in: RITCHIE, B.W.; HARRISON, G.J.; HARRISON, L.R.; Avian medicine: principles and application, p. 641, 1994.

MALUENDA, A.C.H.; CASAGRANDE, R.A.; KANAMURA, C.T.; TORRES, L.N.; NETO, F.G.; GOMES, M.S.; MATUSHIMA, E.R.; Rhabdomyosarcoma in a Yellow-Headed Caracara (Milvago chimachima), Avian Diseases, v. 54, p. 951-954, 2010.

MANAROLLA, G.; RADAELLI, E.; PISONI, G.; SIRONI, G.; RAMPIN, T.; Rhabdomyosarcoma of the pectoral muscles of a free-living European Robin (Erithacua rubecula), Avian Pathology, v. 37, n. 3, p. 311-314, 2009.
MARTINES, N.S.; MACHADO, A.H.A.; SILVA, N.S.; TEDESCO, A.C.; ZÂNGARO, A.; SOARES, C.P.; Avaliação de células neoplásicas após terapia fotodinâmica. Arquivos Catarinenses de Medicina, v. 36, n. 1. 2007.

MCCAW, D.L.; BRYAN, J.N.; In : HENRY, C.J.; Higginbotham, Cancer management in small animal practice, p. 163-166, 2010.

MILLER, R.E.; TRAMPEL, D.W.; BOEVER, W.J.; KLING, M.A.;Carcinoma in the casque of a Greater Indian hornbill (Buceros bicornis); J. Zoo. An. Med. v. 16, p. 131-136, 1985.

REECE, R.L.; Observations on naturally occurring neoplasms in birds in the state of Victoria, Australia, Avian Pathology, v. 21, n. 1, p.3-32, 1992.

ROSENTHAL, K.; DUDA, L.; IVES, E.S.; HAHN, S.M.; A report of photodynamic therapy for squamous cell carcinoma in a cockatiel. Proc. Assoc. Avian. Vet. p. 175-176, 2001.

SEVERINO, D.; JUNQUEIRA, H.C.; GUGLIOTTI, M.; BABRIELLI, D.S.; Baptista,M.S.; Influence of negatively charged interfaces on the ground and excited state properties of methylene blue; Photochemistry and Photobiology, n. 77; p. 459-468; 2003.

SIMPLICIO, F.I.; MAICONCHI, F.; Hioka,N.; Terapia fotodinâmica: aspectos farmacológicos, aplicações e avanços recentes no desenvolvimento de medicamentos. Quimica Nova, v. 25, n. 5, p. 801- 807, 2002.

SINHORINI, J.A.; Neoplasias em aves domésticas e silvestres mantidas em domicílio: Avaliação anatomopatológica e imunoistoquímica, Dissertação de mestrado. Faculdade de Medicina Veterinária e Zootecnia de São Paulo, 2008.

SUEDMEYER, W.K.; HENRY, C.; McCAW, D.; BOUCHER, M.; Attempted photodynamic therapy against patagial squamous cell carcinoma in an African rose-ringer parakeet (Psittacula krameri); J. Zoo. Wildl. Med., v. 38, n. 4, p. 597-600, 2007.

SUEDMEYER, W.K.; McCaw, D.; TURNQUIST, S.; Attempt cell carcinoma in the casque of a Great hornbill (Buceros bicornis), J. Avian Med. Surg, v. 15, n.1, p. 44-49, 2001.

TARDIVO, J.P.; DEL GIGILO,A.; PASCHOAL,L.H.C.; ITO, A.S.; BAPTISTA, M.S.; Treatment of melanoma lesions using methylene blue and RL50 light sourse; Photodyagnosis and Photodynamic Therapy, 2002.

TRAVASSOLY, A.; Rhabdomyosarcoma in a Budgerigar (melopsittacus undulates)- The first case from Iran, Arch. Razi. Ins, v. 52, 2001

WITHROW, S.J.; MaCEWEN, E.G. (Eds.). Small Animal Clinical Oncology. $4^{\text {th }}$ edition. Philadelphia: Saunders, Elsevier, 2007. $846 \mathrm{p}$. 